\title{
La réforme du mariage dans la communauté anabaptiste de Münster : quelle utopie?
}

\section{Catherine DEJEUMONT}

\section{(2) OpenEdition}

12 Journals

\section{Édition électronique}

URL : https://journals.openedition.org/clio/3802

DOI : $10.4000 /$ clio.3802

ISSN : 1777-5299

Éditeur

Belin

Édition imprimée

Date de publication : 1 novembre 2006

Pagination : 27-57

ISBN : 2-85816-867-9

ISSN : $1252-7017$

Référence électronique

Catherine DEJEUMONT, «La réforme du mariage dans la communauté anabaptiste de Münster : quelle utopie? », Clio. Histoire, femmes et sociétés [En ligne], 24 | 2006, mis en ligne le 01 décembre 2008, consulté le 22 avril 2022. URL : http://journals.openedition.org/clio/3802 ; DOI : https://doi.org/ 10.4000/clio.3802

Ce document a été généré automatiquement le 22 avril 2022.

Tous droits réservés 


\title{
La réforme du mariage dans la communauté anabaptiste de Münster : quelle utopie?
}

\author{
Catherine DEJEUMONT
}

1 Les présentes réflexions résultent du croisement de deux questions qui m'ont été posées à plusieurs mois de distance; la première: "Mais vous n'avez pas parlé des femmes ?! » intervenait à l'issue d'un exposé sur l'identité des Schwärmer et des Täufer enthousiastes et anabaptistes -, acteurs éphémères de la Réforme dans le monde germanique; la seconde portait sur les anabaptistes de Münster comme fondateurs d'une utopie sexuelle, demandant des précisions et attendant implicitement une confirmation. Afin de mieux cerner ces deux questions, je me propose dans ce qui suit de situer rapidement l'épisode de Münster dans l'histoire de la Réforme et par rapport à ce que l'on appelle l'anabaptisme, puis d'examiner la thématique du mariage telle qu'elle est définie dans la Restitution ${ }^{1}$ de Bernhard Rothmann, pour ensuite questionner l'historiographie sur ces deux grands thèmes.

L'anabaptisme dans l'histoire de la Réforme

2 Ce que l'on appelle " anabaptisme » est en fait un mouvement complexe, multiforme et polygénétique $^{2}$, dont la diversité est déjà reconnue par les contemporains ${ }^{3}$. Cette désignation est appliquée à tous ceux qui, refusant le baptême des enfants, préconisent et pratiquent le baptême d'adultes conscients de leur foi ; cet acte vaut alors comme adhésion volontaire à l'Église, revendiquée dès lors comme seule Église authentique, ses membres se considérant comme seuls vrais chrétiens ${ }^{4}$. Dans le cadre de la Réforme, la validité du baptême des enfants avait déjà été problématisée par Andreas Carlstadt d'une part et Thomas Müntzer d'autre part, dont l'influence comme inspirateurs de ces divers mouvements est encore reconnue, même si la filiation directe est aujourd'hui contestée $^{5}$. Si l'on reconnaît à présent plusieurs foyers ayant donné naissance à l'anabaptisme ${ }^{6}$, principalement en Suisse et dans le sud de l'Allemagne, c'est à Zurich, en dissidence de la Réforme officielle menée par Huldrych Zwingli, qu'il se manifeste en premier, à partir de 1524, puis avec les premiers baptêmes d'adultes en 1525. Toutefois, 
si le baptême est l'enjeu le plus visible, ce mouvement de protestation implique bien d'autres facteurs, comme la séparation de l'Église et de l'État, la fiscalité, le rapport à l'autorité, la séparation des "vrais" chrétiens du reste de la société, à travers notamment le refus de prêter serment, celui de porter des armes, celui d'assumer des responsabilités publiques, celui des mariages mixtes entre "vrais» et «faux" chrétiens, celui de la propriété privée, et l'exigence d'une éthique bien plus rigoureuse que celle qui se pratiquait alors. Il rassemble d'une manière générale ceux que la Réforme « officielle " a déçus, qui aspirent à des transformations plus profondes et que la recherche a pour cela parfois qualifié "d'aile gauche de la Réforme ${ }^{7}$ », ou de "Réforme radicale ${ }^{8}$ ». Ce mouvement de protestation s'étend rapidement tout d'abord dans les territoires de la Suisse (Zurich, Berne, Saint-Gall, Bâle...) et du sud de l'Empire (Waldshut, Ulm, Augsburg, Nuremberg, Strasbourg...), puis vers l'est (Moravie) et, à partir de 1530, vers le nord (Münster) via la Hollande et la Frise. Les éléments cités cidessus y sont plus ou moins affirmés suivant les divers courants. Certains parmi eux se caractérisent par une référence exclusive à la Bible, en faisant une lecture légaliste ${ }^{9}$ : parmi eux, les Frères Suisses, auteurs en 1527 de ce que l'on appelle la Confession de Schleitheim ${ }^{10}$, prônent une séparation rigoureuse du monde, un refus absolu de porter les armes et la pratique de la communauté des biens, tandis que Balthasar Hubmaier ${ }^{11}$, se fait, lui, l'avocat d'une participation aux instances du pouvoir et défend l'usage des armes s'il est justifié par la politique; d'autres en revanche font une large place aux visions et aux rêves, qu'ils considèrent comme des vecteurs de la révélation divine, ou encore aux prophéties; certains défendent des points de vues millénaristes, comme Hans Hut ${ }^{12}$, ancien disciple de Thomas Müntzer, avocat de la séparation du monde et d'un pacifisme limité dans le temps, puisqu'à la fin du monde qu'il prévoit pour la Pentecôte de 1528, les « élus » étaient selon lui chargés d'« exterminer les impies » pour préparer la parousie, ou encore Melchior Hoffman ${ }^{13}$, prophète annonçant l'avènement de la Nouvelle Jérusalem à Strasbourg transformée en théocratie avec l'appui des autorités politiques, et qui sera le principal inspirateur des anabaptistes de Münster ${ }^{14}$. Les événements de Münster

3 À Münster, en Westphalie, c'est à partir de 1531 que la Réforme prend véritablement pied, sous l'impulsion de Bernhard Rothmann ${ }^{15}$. Ce mouvement s'étend rapidement, et en 1532, toutes les églises de la ville offrent des prédications évangéliques. Le conflit qui en résulte entre la ville et l'évêque dont elle dépend trouve une solution dans le $2^{\mathrm{e}}$ traité de Dülmen, conclu en février 1533, qui officialise la coexistence des deux confessions : le culte catholique est maintenu à la cathédrale et dans les monastères, l'évangélisme est autorisé dans les autres églises. Mais la ville compte également un certain nombre d'anabaptistes, dont l'influence s'accroît elle aussi très rapidement. Dès le printemps de 1533, Bernhard Rothmann notamment, en sa qualité de surintendant chargé de rédiger un règlement ecclésiastique, défend dans son projet leur conception du baptême, et au début du mois d'août est organisée une dispute portant entre autres sur ce point. Rothmann est également co-signataire de la Profession de foi sur le baptême et l'eucharistie ${ }^{16}$, publiée en octobre 1533, texte considéré comme la profession de foi des anabaptistes locaux et qui trouvera un certain écho. À partir du mois d'août, le Conseil de la ville s'efforce en vain d'expulser les prédicateurs anabaptistes ${ }^{17}$. Au début de janvier 1534 ont lieu les premiers baptêmes d'adultes en masse, et l'arrivée de prédicateurs melchiorites ${ }^{18}$ en provenance de Frise et de Hollande accentue encore la radicalisation en cours et les troubles dans la ville. L'élection du 23 février 1534 donne aux anabaptistes la majorité des sièges au conseil de la ville. Ils passent alors d'une 
semi-clandestinité à l'exercice du pouvoir et à l'action. Münster est assiégée par l'évêque Franz von Waldeck, allié à certains princes protestants, siège qui durera jusqu'à la prise de la ville, fin juin 1535.

4 Pendant cette période, dans la ville de Münster, les structures politiques seront transformées en profondeur. Le conseil est tout d'abord maintenu, mais placé sous l'autorité du prophète Jan Matthijs ${ }^{19}$, dont il ne fait plus qu'entériner les décisions ${ }^{20}$. Il ne s'agit plus de se séparer du monde, mais, compte tenu de la fin de celui-ci annoncée pour le 5 avril (jour de Pâques) 1534, de préparer la parousie en exterminant les «impies ». Ceux qui refusent le baptême sont menacés de mort et contraints à l'exil, leurs biens sont confisqués ${ }^{21}$. La communauté des biens est instituée, les échanges monétaires supprimés ${ }^{22}$, les documents à caractère juridique ainsi que les livres, à l'exception de la Bible ${ }^{23}$, sont jetés au feu. Après la mort de Jan Matthijs lors d'une tentative pour briser le siège le 5 avril, ses fonctions sont reprises par Johan Bockelson, dit Jan von Leiden ${ }^{24}$, qui procède à d'autres transformations. Münster devient officiellement la Nouvelle Jérusalem, gouvernée par le Conseil des Anciens des douze tribus d'Israël placé sous son autorité de prophète, puis, à partir de septembre 1534, une théocratie dont il est le roi ${ }^{25}$. Parmi d'autres réformes illustrant la volonté de rompre avec le passé26, le mariage lui-même est transformé, puisqu'il devient obligatoire pour tous, hommes et femmes, sous la forme spécifique de la polygamie ${ }^{27}$. De toutes ces mesures, c'est sans doute cette dernière, instituée officiellement le 23 juillet 1534, qui suscitera le plus de réactions et de polémiques, à la fois à Münster même, puisqu'elle sera à l'origine d'une révolte une semaine plus $\operatorname{tard}^{28}$, et à l'extérieur, parmi les contemporains comme pour la postérité29.

Le mariage dans la Restitution de Bernhard Rothmann

Publiée en octobre 1534, la Restitution rédigée par Bernhard Rothmann, devenu porteparole officiel du régime, est particulièrement intéressante : dans cette ville assiégée et soumise à des tensions internes, l'enjeu est de justifier les mesures prises, de désavouer la propagande adverse et de convaincre pour gagner des partisans. Ce texte s'inscrit en effet dans le «dialogue » assez fourni mené pendant seize mois entre assiégeants et assiégés ${ }^{30}$ par-dessus les fortifications et est considéré à juste titre comme le " programme » de la communauté de Münster ${ }^{31}$. Rédigé en bas-allemand, il a fait l'objet de deux éditions, a été également distribué aux Pays-Bas, et il a donné lieu pratiquement immédiatement à un certain nombre de réfutations ${ }^{32}$. Il s'agit d'un traité assez volumineux, puisqu'il compte 64 feuillets dans la première édition, 48 dans la seconde ${ }^{33}$, et se compose de dix-huit chapitres développant le thème du rétablissement de la véritable doctrine chrétienne annoncé dans le titre, placés sous le signe de la restauration universelle annoncée dans les Actes des Apôtres ${ }^{34}$. Après avoir exposé la nécessité de celle-ci ${ }^{35}$, il traite donc d'un certain nombre de points fondamentaux comme l'Écriture ${ }^{36}$, la christologie ${ }^{37}$, le baptême ${ }^{38}$, la définition de l'Église ${ }^{39}$, le librearbitre et le péché ${ }^{40}$, la communauté des saints ${ }^{41}$, l'eucharistie $e^{42}$, la parousie et le règne du Christ sur terre ${ }^{43}$, l'usage de la force par les autorités politiques (chrétiennes) ${ }^{44}$, et le mariage, auquel sont consacrés trois chapitres ${ }^{45}$. Le premier d'entre eux s'attache à la définition $\mathrm{du}$ "véritable mariage chrétien $»^{46}$, dont la notion centrale est celle de la fécondité, ancrée dans le texte de la Genèse : «soyez féconds, multipliez, emplissez la terre et soumettez-la $»^{47}$. Cette fécondité, qui est à la fois une bénédiction et un don de Dieu, est progressivement ré-interprétée comme une prescription ${ }^{48}$ donnée par Dieu à tous les hommes et devant donc être respectée à la lettre. Couplé dans cette 
argumentation à un extrait de l'Épître aux Hébreux rappelant: «Que le mariage soit honoré de tous et le lit nuptial sans souillure $»^{49}$, le mariage est donc rétabli dans son caractère sacré, caractère garanti par les interdictions formulées également par Dieu. Ce chapitre présente ensuite un certain nombre d'interdits rappelant ceux du Lévitique: interdiction de l'adultère, car il est improductif «et pour d'autres raisons $\aleph^{50}$, de la 'fornication', elle aussi improductive et ne visant que la recherche des plaisirs de la chair, tout comme l'union avec une femme enceinte ou stérile, interdiction de l'union "contre nature, bestiale et infâme " ${ }^{51}$, et rappel que sont considérés comme des péchés le fait de «brûler seul» et la "souillure pendant le sommeil $\aleph^{52}$. Enfin, l'injonction de l'Épître aux Hébreux ne pouvant être respectée que si les deux époux « craignent Dieu » et «ont une foi pure », le mariage véritable ne peut exister qu'entre "vrais chrétiens ${ }^{53}$. Ce dernier volet de l'argumentation permet de déclarer nuls les mariages conclus avant le «renouvellement» des croyants par le nouveau baptême, ceux qui le souhaitent devant conclure un nouveau mariage ${ }^{54}$, les autres étant tenus de se marier sans tarder. Le chapitre suivant est consacré à l'homme, à ce qui est convenable pour lui et à sa majesté dans le mariage ${ }^{55}$. Rappelant que Dieu a créé l'humanité pour le mariage, Rothmann re-précise la hiérarchie fondamentale de l'univers, instituée par Dieu, qui soumet l'homme à Dieu et la femme à l'homme, rappelant que «l'homme ne doit écouter personne d'autre que Dieu, afin d'éviter d'être trompé, comme lorsqu'il a écouté la femme ${ }^{56}$ » et que «l'homme a reçu de Dieu le pouvoir d'être un seigneur et un maître pour sa femme, tout comme Dieu est un seigneur et un maitre pour lui ${ }^{57}$ ». L'homme doit donc : craindre Dieu et observer sa loi ; prendre une femme vivant elle aussi «dans la crainte de Dieu, calme et douce de caractère $~^{58}$; être un seigneur et un chef pour son épouse, comme Christ est un seigneur et un chef pour son Église ${ }^{59}$, c'est-à-dire la dominer, rechercher par elle une descendance pour la gloire de Dieu, et l'instruire, l'assister, la guider, et le cas échéant la punir si elle manque à son devoir conjugal d'obéissance (il est en droit de la quitter si elle est trop " personnelle $"^{60}$ ) ; et surtout : se préserver de toute souillure et de tout mauvais usage de ce don de Dieu qu'est sa semence. Or, pour un homme que Dieu a richement doté, capable de féconder plus d'une femme, il est impossible de respecter ce dernier point tant qu'il ignore la «magnifique liberté de l'homme dans le mariage " qu'il convient de rétablir. Cette liberté, c'est celle d'avoir plus d'une épouse ${ }^{61}$, et c'est même une nécessité, étant donné que le concubinage, l'adultère, etc. ${ }^{62}$ sont interdits. La Bible en témoigne avec les exemples d'Abraham ou de David, entre autres. La polygamie n'est pas interdite par l'Écriture, et l'Ancien Testament vaut bien sûr autant, sinon plus, que le Nouveau, poursuit Rothmann. Ce chapitre se termine sur un certain nombre de répétitions relatives à la liberté de l'homme, à ses "chastes besoins " ${ }^{63}$, à son statut de seigneur et maître, et à l'impératif absolu de préserver la pureté de son mariage, concluant que « ce sont les femmes qui dominent presque partout, menant les hommes comme on mène les ours, et [que] le monde entier est noyé dans l'adultère, la souillure et la fornication ${ }^{64} »$. Le chapitre suivant, consacré à la femme, à ce qui est convenable pour elle et à sa soumission dans le mariage ${ }^{65}$, est beaucoup plus court, puisqu'il équivaut en volume à environ un cinquième du précédent. Il commence par une mise au point, rendue nécessaire par le fait, nous dit le texte, que le monde fonctionnait jusqu'alors à l'envers ${ }^{66}$ : dans le mariage, la femme doit être féconde et être soumise à l'homme - cette seconde injonction valant également pour celles qui «n'étant pas féminines, ne peuvent servir à l'homme pour l'usage naturel ${ }^{67}$. Suit un nouveau rappel de la hiérarchie Dieu - Christ - homme - femme, qui souligne le 
pouvoir de décision absolu de Dieu et le fait que l'homme est l'image de Dieu. De même que le Christ s'en remet à la volonté de son Père, l'homme est soumis en toute obéissance au Christ; la femme elle aussi doit donc «avoir toujours le regard fixé sur son mari, qui est son seigneur et maître, et cela sans grogner ni contredire celui-ci ${ }^{68}$. Ce chapitre reprend, beaucoup plus rapidement, une partie de l'argumentation développée précédemment, en soulignant avec insistance la répartition édictée par Dieu : le pouvoir de décision est et doit être détenu et exercé par Dieu et donc par l'homme ${ }^{69}$, la femme n'y ayant aucune part, mais devant se contenter d'obéir en silence $^{70}$. Le ton est ici nettement plus polémique qu'auparavant, les hommes y étant incités à faire "plier les femmes, qui portent maintenant la culotte un peu partout, pour les ramener à l'obéissance $»^{71}$ et à " cesser d'être des femmes ${ }^{72}$ (!). Le chapitre se termine sur un accent de triomphe, affirmant que la restauration promise par Jérémie ${ }^{73}$ est une réalité à Münster, et que Dieu y a « désormais soumis toutes les femmes aux hommes ", que «la parole divine a fait que toutes, les jeunes comme les vieilles, sont contraintes d'accepter d'être régies par l'homme $»^{74}$, et appelant les hommes à "se réveiller $»^{75}$.

6 Cette présentation volontairement détaillée des chapitres consacrés au mariage dans la Restitution aura sans doute permis au lecteur d'ébaucher lui-même des réponses aux questions formulées au début de ces réflexions. Il n'est sans doute pas sans intérêt de rappeler d'une part que ce qui est exposé précédemment se distingue de textes contemporains évidemment par sa défense de la polygamie, mais aussi par la violence de ton qui marque ici les relations entre hommes et femmes; en revanche, l'option fondamentale, elle, qui assujettit la femme à l'homme, n'est pas ressentie comme particulièrement choquante ${ }^{76}$, la définition du mariage par la procréation rappelant la position traditionnelle de l'Église à partir de Saint Augustin, tandis que la reconnaissance de la puissance de la sexualité (masculine) rappelle certains développements de Martin Luther ${ }^{77}$, et d'autre part, que ce qui peut apparaitre comme un projet de société n'en est pas un, puisqu'il s'agit de l'élaboration théorique légitimant a posteriori des réformes entrées en vigueur précédemment, et manifestement contestées. La révolte suivant l'introduction de la polygamie a donné lieu à une répression spectaculaire à laquelle a participé une partie de la population ${ }^{78}$, mais, en dépit des pressions exercées sur les femmes (tentatives d'intimidation, incarcérations, exécutions), la résistance est cependant telle qu'à la fin de l'été, il leur est accordé, pendant un bref délai, la possibilité de demander le divorce si elles peuvent prouver qu'elles ont été mariées sous la contrainte - droit dont font usage une centaine d'entre elles ${ }^{79}$. Il sera par la suite rappelé publiquement que le mariage doit se faire par consentement mutuel. La publication de ce texte est postérieure à ces événements. Celui-ci n'est donc pas à proprement parler novateur, et présente une cohérence indéniable avec certaines des lois édictées par les régimes successifs : dès le mois d'avril, le Conseil des Anciens des douze tribus d'Israël avait promulgué un

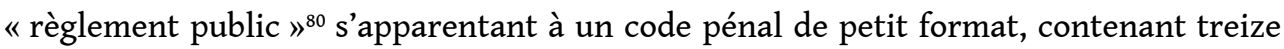
articles encadrés par un préambule et une conclusion plaçant l'ensemble de cet appareil sous l'autorité de la Bible et annonçant la peine capitale pour les crimes mentionnés dans ces treize catégories. On trouve parmi ceux-ci, à l'article 3 : la désobéissance de la femme envers son mari, à l'article 5 : l'adultère, à l'article 6 correspondant dans l'ensemble aux proscriptions édictées dans la Restitution -: la 'fornication', le viol (de femmes et d'hommes), l'« impudicité », les relations sexuelles entre membres d'une même parentèle, et les relations sexuelles avec une femme 
pendant sa menstruation ${ }^{81}$. L' «Artikelbrief» du 2 janvier $1535^{82}$ est moins détaillé à cet égard, puisqu'il ne retient plus que l'adultère, la "fornication $»^{83}$ et le viol ${ }^{84}$ comme crimes; il prévoit en revanche qu'une femme dont le mari n'a pas été vu depuis trois nuits peut (et doit) se remarier ${ }^{85}$. La mise en perspective de la Restitution et de ces textes juridiques permet de comprendre qu'il ne s'agit à Münster ni de liberté sexuelle, ni pour l'homme, ni (surtout pas) pour la femme, ni de pratiquer le «communisme des femmes ${ }^{86}$. Loin d'être affaibli ou supprimé, le mariage y est ré-affirmé comme le fondement de la société, sous une forme élargie, puisqu'il ne s'agit plus à proprement parler de couples, mais de groupes, placés sous l'autorité de l'homme. Les relations entre hommes et femmes n'y sont aucunement « révolutionnées ", mais, au contraire, fixées à nouveau de façon implacable sur le modèle du patriarcat de l'Ancien Testament. On peut certes expliquer ce choix, ou du moins tenter de le faire en rappelant les circonstances spécifiques à cet îlot qu'est alors la 'communauté des saints' de Münster, qui regroupe dans un territoire fermé environ 2000 hommes et 5000 femmes ${ }^{87}$, dont de nombreux étrangers venus (parfois seuls ${ }^{88}$ ) de Frise ou de Hollande, un certain nombre de femmes restées à Münster pour veiller sur les biens de leur famille plus que par conviction ${ }^{89}$, et une partie des habitants qui, effrayés par le spectacle de ce qui attend ceux qui, quittant la ville, sont arrêtés ou exécutés par les assiégeants après avoir été humiliés, molestés et dépossédés par les assiégés, préfèrent rester sur place ${ }^{90}$ - population qu'il convient de lier en une véritable communauté, de contrôler sur le plan politique et du point de vue de la moralité publique. Pour cela, la foi n'étant manifestement pas un ciment suffisant, c'est à la terreur que ces régimes successifs auront recours. Un autre élément, développé de façon convaincante par Matthias Hennig91, peut compléter cette tentative d'explication: c'est le rigorisme moral revendiqué par les anabaptistes - ceux de Münster ${ }^{92}$ comme d'autres groupes comme une partie de leur identité les distinguant non seulement des catholiques, mais également des luthériens et zwingliens. La réforme du mariage à Münster ne résultait pas, conclut-il, d'une volonté de libération de la sexualité mais d'une tentative faite pour la réglementer ${ }^{93}$, prenant en compte les aspects 'trop humains' des membres de cette communauté ${ }^{94}$. Pour cet auteur, cette tentative n'est pas «tout à fait réussie », et il est l'un des rares, parmi les historiens modernes, avec James M. Stayer ${ }^{95}$, à faire remarquer que ce modèle n'était pas très favorable aux femmes, concluant qu'il ne contient rien qui puisse permettre de l'interpréter comme une «forme particulièrement émancipatrice de la vie en communauté ${ }^{96}$. Il semble en effet difficile de soutenir ce point de vue et de le présenter comme un modèle souhaitable. En guise de conclusion intermédiaire, on peut donc faire remarquer que le sort réservé à la femme à Münster semble peu enviable, et que le terme 'utopie' apparaît peu adapté pour désigner un modèle de société qui n'est ni novateur, ni idéal, ni imaginaire, puisqu'il a été en vigueur pendant près d'un an; on peut par ailleurs se demander si cette 'utopie' n'existe pas plutôt uniquement dans l'esprit des tiers, interprétant cette réalité au gré de leur propre subjectivité, voire de leurs intérêts.

Münster et ses interprétations

7 On peut également se demander d'où vient cette aura sulfureuse qui s'attache à Münster, et il faut pour y répondre se pencher sur l'historiographie. Par un phénomène similaire à celui qui veut que pour avoir une idée de la situation des femmes à Münster, il faut interroger les hommes - les seuls qui aient laissé des traces écrites (et il est à cet égard saisissant de constater combien, dans le chapitre officiellement consacré aux femmes dans la Restitution, il est toujours question des hommes) ${ }^{97}-$, on ne connaît 
souvent Münster, en effet, qu'à travers ce que d'autres en ont dit. Les écrits de Bernhard Rothmann sont peu connus. En revanche, les textes des détracteurs de l'anabaptisme ont connu une assez large diffusion. Ce sont donc en grande partie leurs adversaires qui, dans un premier temps du moins, ont «écrit l'histoire $"^{98}$, celle de Münster s'intégrant dans l'histoire de l'anabaptisme, qui se construira en plusieurs étapes : d'abord au XVI ${ }^{e}$, puis au XIX', puis au XXe siècles.

$8 \mathrm{Au} \mathrm{XVI}^{\mathrm{e}}$ siècle, si les anabaptistes ont en effet assez peu écrit et n'avaient qu'un accès limité à l'imprimerie, leurs adversaires en revanche, écrivent, publient et diffusent dès 1525 un portrait qui, progressivement affiné (et faussé) au gré des circonstances, les suivra, de la même façon que la répression accompagne la propagation de leurs idées. Sous la plume de Zwingli, puis d'Oecolampade, d'Urbanus Rhegius, de Johannes Fabri, de Martin Luther, de Melanchthon entre autres, la même argumentation se retrouve : hérétiques, schismatiques, blasphémateurs, ils mettent en péril la chrétienté; ils sont accusés de ne respecter ni les autorités, ni la propriété privée, ni le mariage, fondements de la société telle qu'elle est voulue par Dieu et que les théologiens s'attacheront à défendre ; la rigueur morale qu'ils affichent est tournée en dérision et dénoncée comme la pire manifestation de l'hypocrisie ; enfin, l'engagement de certains anabaptistes dans la guerre des paysans ${ }^{99}$ permet de les dénoncer comme de dangereux agitateurs, fauteurs d'insurrection, constituant donc un péril politique pour l'Empire ${ }^{100}$. Criminalisés et passibles de la peine de mort dès 1525 à Zurich, ils le seront au niveau de l'Empire par l'édit de Spire de 1529, résultat de l'union des catholiques et des " protestants ». Ces derniers se démarquent à nouveau officiellement des anabaptistes dans la Confession d'Augsbourg de 1530. C'est dans ce contexte qu'interviennent les événements de Münster. Les réactions officielles notamment à la Restitution de Bernhard Rothmann obéissent à la même logique et reprennent dans ses grandes lignes la même argumentation ${ }^{101}$. Après la chute de Münster, les textes relatant les événements sont assez nombreux ${ }^{102}$. Ici encore, les luthériens, accusés d'avoir été à l'origine des troubles qui y ont commencé avec l'introduction de la Réforme, s'en défendent. Le texte intitulé « Histoire véridique des débuts de l'évangélisme à Münster, puis de sa fin, causée par les anabaptistes ", publié en 1536 à Wittemberg et préfacé par Bugenhagen, fera autorité ${ }^{103}$. Il est l'œuvre d'un certain Heinrich Dorpius, pseudonyme sous lequel la recherche tend aujourd'hui à voir Antonius Corvinus, théologien luthérien attaché à Philippe de Hesse et ayant participé au procès de Jan von Leiden, Bernhard Knipperdolling et Bernhard Krechting ${ }^{104}$. Manifestement bien documenté, il mêle récit et polémique, n'oubliant pas d'accuser l'Église catholique, ni de présenter le camp évangélique (c'est-à-dire les luthériens) sous son meilleur jour, ni d'insister sur les discordances entre le discours des anabaptistes et leurs actes: blasphémateurs, insurgés, violents voire barbares, voleurs, menteurs, débauchés, etc., ils ont mérité leur châtiment, expression de la colère divine. Leur destin doit servir d'exemple. Cette " Histoire véridique » est citée explicitement par d'autres auteurs postérieurs, comme Justus Menius et Heinrich Bullinger. Proche de Martin Luther, qui préface son texte, Justus Menius publie une dizaine d'années plus tard une étude plus générale intitulée "De l'esprit des anabaptistes » ${ }^{105}$. C'est sans doute par ce texte qu'est fixé, avec l'assimilation des anabaptistes de Münster à Thomas Müntzer ${ }^{106}$, mort en mai 1525 à l'issue de la guerre des paysans, un élément supplémentaire dans l'argumentation ${ }^{107}$. Cet amalgame restera dans l'histoire : il réapparaît vers la fin $\mathrm{du} \mathrm{XVI}^{\mathrm{e}}$ siècle sous la plume de Heinrich Bullinger ${ }^{108}$, successeur de Zwingli à Zurich, puis, inversé, faisant de Müntzer un anabaptiste, sous celle de Friedrich Engels ${ }^{109}$ comme, plus récemment, sous 
celle de Paul Ricœur ${ }^{110}$. Jusqu'à la fin du XVI ${ }^{e}$ siècle, les enjeux politico-religieux imposent aux "survivants » de la Réforme, catholiques comme réformés, de dresser un portrait exclusivement négatif des anabaptistes, les présentant tous à la lumière de ce qu'ils veulent retenir de l'épisode de Münster: un grand nombre de morts, un grand désordre social, un grand péril pour la propriété privée et pour les bonnes mœurs polarisation qui permet aux catholiques de dénoncer la Réforme comme l'origine de tout le mal, et aux réformés d'insister sur leur grand respect de l'ordre politique et de l'ordre social. À cet égard, si du point de vue des «bonnes mœurs", les faits relatés semblent en effet difficilement conciliables avec les critères moraux officiellement affichés, ils relèvent sans doute plus des circonstances dans cette cité assiégée que d'une volonté des autorités locales - la guerre crée des circonstances peu propices à l'éclosion d'une grande rigueur morale, mais le programme dressé par les autorités relève d'une autre dimension. Or, le cadre fixé par les autorités de Münster, en matière de mariage, n'est pas celui d'une très grande permissivité. La pratique du «communisme des femmes» si fréquemment évoquée, et contre laquelle Bernhard Rothmann lui-même proteste dans un texte ${ }^{111}$ publié après l'entrée en vigueur de la communauté des biens, mais avant celle de la polygamie, relève ainsi de la propagande ; elle exploite en effet le phénomène très minoritaire et très éphémère de communautés anabaptistes libertines ${ }^{112}$ ayant effectivement existé en 1525 à Appenzell et à SaintGall ${ }^{113}$ pour discréditer l'ensemble des anabaptistes, tout comme la participation de certains d'entre eux à la guerre des paysans a été exploitée pour les présenter tous comme de dangereux insurgés. Les commentaires élaborés par le camp catholique obéissent à cette même logique et à la fin du XVI ${ }^{\mathrm{e}}$ siècle, l'image de l'anabaptisme est donc essentiellement négative.

9 La deuxième grande étape dans la construction de cette image interviendra au milieu du XIXe siècle, après les espoirs déçus par l'échec de la révolution de 1848. C'est à cette époque que Carl Adolf Cornelius publie sa grande étude sur les événements de Münster, ainsi qu'un certain nombre de sources inconnues jusqu'alors, comme le récit du menuisier Heinrich Gresbeck ${ }^{114}$, qui occupe une position particulière à plusieurs titres. C'est en effet le seul témoignage de première main de cette ampleur parvenu jusqu'à nous, puisque Gresbeck, catholique, rendant visite à sa mère, se trouve piégé par les événements et vit donc à Münster jusqu'à son évasion et sa trahison à la fin du mois de mai 1535 ; c'est en effet apparemment grâce à lui que la ville est tombée aux mains des troupes de l'évêque - épisode occulté dans la version de Dorpius, qui mentionne bien le traître comme un « envoyé de Dieu », mais cite un autre nom. Il semble avoir rédigé ces souvenirs une dizaine d'années après les faits, et, bien que partial, il est qualifié de "très fiable» par Cornelius ${ }^{115}$. Ce récit très humain et touchant recoupe parfois la présentation des faits par Dorpius, mais également certains événements relevés notamment par Urbanus Rhegius ou par Philippe de Hesse, témoignant ainsi de la correspondance qui existait entre les deux partis en présence. Ce texte n'est donc connu que depuis le milieu du XIXe siècle. C'est également à cette époque que Friedrich Engels publie son étude sur la guerre des paysans, dans laquelle il oppose sans nuances Martin Luther asservi aux princes à Thomas Müntzer le héros du peuple, révolutionnaire victime de la répression ${ }^{116}$. L'image de ce dernier devient alors exclusivement positive, revalorisant également celle des anabaptistes auxquels il est explicitement associé par cet auteur. Adoptée par l'historiographie d'inspiration marxiste, en particulier celle de l'ex-RDA, elle persiste et englobe bien sûr, l'épisode de Münster. Les anabaptistes - ceux de Münster comme les autres, tout aussi méconnus - 
trouvent ainsi une image plutôt positive de révolutionnaires écrasés par les forces de la réaction. Leur approche du mariage, critiquée par les communistes ${ }^{117}$, est parfois reinterprétée dans la mouvance de 1968 comme l'expression d'une volonté libertaire, et célébrée comme telle ${ }^{118}$. C'est dans ce courant que s'inscrit leur portrait par Marguerite Yourcenar dans son roman L'Euvre au noir, paru à la fin du mois d'avril $1968^{119}$; salué de façon quasi-unanime comme une œuvre littéraire d'importance et comme un grand roman historique, il a sans aucun doute contribue à propager de cette communauté l'image de doux idéalistes pratiquant l'amour libre, victimes sympathiques de la répression des puissants, qui semble encore être parfois la leur aujourd'hui. Le chapitre consacré à Münster dans ce roman ne mentionne aucunement le mariage, il y est en revanche question de désir et de plaisir, essentiellement féminins - présentation des faits qui peut avoir suffi pour lire quelques années plus tard que «[...] Jean de Leyde [...] instaure l'amour libre [...] $»^{120}$.

10 Enfin, pour revenir à la question des femmes, si l'anabaptisme a pu leur offrir une situation un peu plus favorable que d'autres environnements, traits dégagés par Ernst Troelsch et Max Weber ${ }^{121}$ entre autres, ce n'est sans doute pas dans la communauté de Münster qu'il faut en chercher des traces. À part les passages cités précédemment, la femme apparaît dans la Restitution exclusivement comme celle qui a causé la chute de l'homme $^{122}$. Münster rejoint en cela l'histoire générale de l'anabaptisme et celle de la Réforme : les femmes ont dans l'ensemble très peu écrit ${ }^{123}$, et en l'occurrence pas du tout. S'il y eut à Münster des prophétesses ${ }^{124}$, elles ne comptaient pas parmi les personnages influents des régimes successifs, les principaux prophètes étant des hommes : Jan Matthijs, Jan von Leiden, Bernhard Knipperdolling, Johann Dusentschur. La prophétie semblait en outre être assez commune à Münster, puisque Bernhard Rothmann, dans le dernier chapitre de la Restitution, relatant les 'miracles' survenus à Münster et témoignant de la bienveillance de Dieu à leur égard mentionne un épisode où " tout le monde prophétisait, même les enfants de sept ans $»^{125}$. Cet aspect ne peut donc pas être retenu non plus comme attestant d'une situation plus favorable aux femmes à Münster qu'ailleurs à la même époque.

11 Il peut sembler facile, avec le recul, de porter un jugement sur les événements d'une époque révolue. Il est également facile d'y voir ce qu'on veut y voir : simples fous ${ }^{126}$, précurseurs de révolutionnaires, de libertaires, de libertins ou de l'émancipation de la femme, et cela, d'autant plus si, comme dans le cas présent, on a commencé par réduire au silence les intéressés. Il est plus difficile d'appréhender le monde mental de cette époque, sous-tendu par l'omniprésence de Dieu. Dans ce contexte, tout manquement est réputé attirer la colère divine sur l'individu et / ou le groupe qui en est responsable ou qui le tolère. C'est cette référence universelle qui explique l'importance du blasphème dans l'argumentation religieuse, juridique et pénale de l'époque. C'est bien sûr elle qui justifie également l'organisation du mariage. Dans cette société des débuts des "temps modernes", la notion de "progrès" n'existe pas. Seule compte l'adéquation à la volonté divine, d'où l'importance accordée à la juste compréhension et à la juste interprétation de celle-ci, chaque camp revendiquant ainsi la vérité à son profit $^{127}$. Cette question du mariage n'est qu'un des aspects des réflexions qui touchent à l'époque pratiquement tous les aspects de la société et de la vie. Le débat sur la polygamie notamment se poursuivra au-delà de la chute de Münster ${ }^{128}$ où le modèle élaboré n'était, on l'a vu, ni très permissif, ni très favorable aux femmes. Cependant, par-delà les maigres correctifs relevés dans ce qui précède, plutôt qu'à une recherche de «parrains » hypothétiques dans l'histoire, c'est à un retour sur nous-mêmes que 
cette étude pourrait et devrait nous inciter. En dépit de ce que la société dans l'Allemagne de la Réforme peut avoir d'étranger pour nous, certaines des questions de cette époque nous sont, elles, familières : celle par exemple de la définition du mariage, celle des aspirations à des repères moraux, ou celle du terrorisme religieux. Quelles réponses pouvons-nous y apporter? Quel lien unit de façon aussi nette la notion de propriété privée et celle du mariage ? Enfin, si la société de Münster était ancrée dans le religieux, qu'en est-il de la nôtre: est-elle aussi profondément laïque qu'elle veut bien le dire ? Sommes-nous aussi loin que nous l'affirmons du classique « c'est la femme qui... ", réponse d'Adam à Dieu, et de l'homme image de Dieu et détenteur de tout pouvoir $^{129}$ ? Autant de questions qui sont autant de pistes de réflexions sur nousmêmes.

\section{BIBLIOGRAPHIE}

BAUTZ Friedrich Wilhelm, 1990a, « Johann Bockelson », in Biographisch-Bibliographisches Kirchenlexikon, I, Hamm, Bautz, p. 641-642.

--, 1990b, « Balthasar Hubmaier », in Biographisch-Bibliographisches Kirchenlexikon, II, Hamm, Bautz, p. 1109-1114.

--, 1990c, « Melchior Hoffmann », in Biographisch-Bibliographisches Kirchenlexikon, II, Hamm, Bautz, p. $974-978$.

BERNET Claus, 2003, « Jan Matthijs », in Biographisch-Bibliographisches Kirchenlexikon, XXI, Nordhausen, Bautz, p. 912-916.

BEZE Théodore de, 1568, Tractatio de polygamia et divortiis, Genève.

BLICKLE Peter, 1993, Die Revolution von 1525 (3éd.), München, Oldenbourg Verlag.

BOURDIEU Pierre, 1998, La domination masculine, Paris, Seuil.

BULLINGER Heinrich, (1560) 1983, « Der Widertöufferen Ursprung », in Robert Stupperich (éd.), Die Schriften der münsterischen Täufer und ihrer Gegner, III. Schriften von evangelischer Seite gegen die Täufer, Münster, Aschendorffsche Verlagsbuchhandlung, p. 248-265.

BRANT Sebastian, (1494) 1977, La Nef des Fous, Strasbourg, éd. de la Nuée Bleue, (trad. : Madeleine Horst, $2^{e}$ éd., 2005).

CHAIX Gérald, 2002, «Les anabaptistes de Münster (1534-1535) : Bilan historiographique », Bulletin de la Société d'Histoire du Protestantisme Français, 148/2, p. 293-307.

CORNELIUS Carl Adolf (éd.), 1853, Berichte der Augenzeugen über das münsterische Wiedertäuferreich, Münster, Theissing.

--, 1855, 1860, Geschichte des Münsterischen Aufruhrs, Leipzig, Weigel Verlag, (2 Vol.).

CONRAD Anne / MICHALIK Kerstin (éd.), 1999, Quellen zur Geschichte der Frauen, Bd. 3. Neuzeit, Stuttgart, Reclam. 
DEJEUMONT Catherine, 2002, “"Schwärmer”, “Geist”, “Täufer”, “Ketzer” : de l'allié au criminel (1522-1550) », Bulletin de la Société d'Histoire du Protestantisme Français, 148/1, p. 21-46.

DEPPERMANN Klaus, PACKULl Werner O., STAYER James M., 1975, « From Monogenesis to Polygenesis. The Historical Discussion of Anabaptist Origins », Mennonite Quarterly Review, 49, p. 83-121.

DEPPERMANN Klaus, 1979, Melchior Hoffmann. Soziale Unruhen und apokalyptische Visionen im Zeitalter der Reformation, Göttingen, Vandenhoeck \& Ruprecht.

DETMER Heinrich (éd.), 1900, Die Geschichtsquellen des Bistums Münster, V-VI, Münster, Theissing.

--, 1903, Bilder aus den religiösen und sozialen Unruhen in Münster während des 16. Jahrhunderts. I.

Johann von Leiden. Seine Persönlichkeit und seine Stellung im Münsterischen Reiche, Münster,

Coppenrath.

--, 1904, Bilder aus den religiösen und sozialen Unruhen in Münster während des 16. Jahrhunderts. II.

Bernhard Rothmann. Kirchliche und soziale Wirren in Münster 1525-1535. Der täuferische Kommunismus, Münster, Coppenrath.

DORPIUS Henricus, (1536) 1983, « Wahrhafftige historia, wie das Evangelium zu Münster angefangen und darnach durch die Widertäuffer verstört, wider aufgehört hat », in Robert Stupperich (éd.), Die Schriften der münsterischen Täufer und ihrer Gegner, III. Schriften von evangelischer Seite gegen die Täufer, Münster, Aschendorffsche Verlagsbuchhandlung, p. 220-244.

--, 1992, in Adolf Laube (éd.), Flugschriften vom Bauernkrieg zum Täuferreich (1526-1535), Berlin, Akademie Verlag, p. 1662-1697.

ENGELS Friedrich, 1960, « Der deutsche Bauernkrieg », in Karl Marx / Friedrich Engels, Werke, 7, Berlin (DDR), Dietz Verlag, p. 327-413.

FABRI Johannes, (1528a) 1992, « Ursache, warum der Wiedertäufer Patron und erste Anfänger, Doktor Balthasar Hubmaier, verbrannt wurde », in Adolf Laube (éd.), Flugschriften vom Bauernkrieg zum Täuferreich (1526-1535), Berlin, Akademie Verlag, p. 1580-1604.

--, (1528b) 2000, « Sermone wider die gottlosen Wiedertäufer », in Adolf Laube (éd.), Flugschriften gegen die Reformation (1525-1530), Berlin, Akademie Verlag, p. 671-700.

FAST Heinold (éd.), 1962, Der linke Flügel der Reformation. Glaubenszeugnisse der Täufer, Spiritualisten, Schwärmer und Antitrinitarier, Bremen, Carl Schünemann Verlag.

FISCHER Carsten, 2004, Die Täufer in Münster (1534/35) - Recht und Verfassung einer chiliastischen Theokratie, http://www.forhistiur.de/zitat/ 0408fischer.htm.

GOERTZ Hans-Jürgen, 1988, Die Täufer. Geschichte und Deutung, München, Beck.

GRESBECK Heinrich, 1853, "Bericht von der Wiedertauffe in Münster ", in Carl Adolf Cornelius (éd.), Berichte der Augenzeugen über das münsterische Wiedertäuferreich, Münster, Theissing, p. 1-214. GROCHOWINA Nicole, 1999, « Zwischen Gleichheit im Martyrium und Unterordnung in der Ehe. Aktionsräume von Frauen in der täuferischen Bewegung ", in Anne Conrad (éd.), "In Christo ist weder man noch weyb" - Frauen zu der Zeit der Reformation und der katholischen Reform, Münster, Aschendorff, p. 95-113.

HAAS Martin, 1977, « Der Weg der Täufer in die Absonderung », in Hans-Jürgen Goertz (éd.), Umstrittenes Täufertum : 1525-1975 ; Neue Forschungen, Göttingen, Vandenhoeck \& Ruprecht, p. 50-78.

HADDAD Gérard, 1990, Les folies millénaristes - les biblioclastes, Paris, Grasset. 
HAUDE Sigrun, 2000, In the Shadow of "Savage Wolves" : Anabaptist Münster and the German Reformation during the 1530s, Boston / Leiden / Cologne, Humanities Press.

HEINZ Daniel, 1995, « Michael Sattler », in Biographisch-Bibliographisches Kirchenlexikon VIII, Herzberg, Verlag Traugott Bautz, p. 1403-1408.

HENNIG Matthias, 1983, « Askese und Ausschweifung. Zum Verständnis der Vielweiberei im Täuferreich zu Münster 1534/35 », Mennonitische Geschichtsblätter, 35, p. 25-45.

KERSSENBROICK Hermann von, (1575) 1900, « Anabaptistici furoris Monasterium inclitam Westphaliae metropolim evertentis historica narratio », in Heinrich Detmer (éd.), Die Geschichtsquellen des Bistums Münster, V-VI, Münster, Theissing.

KIRCHHOFF Karl-Heinz, 1989, « Das Phänomen des Täuferreiches zu Münster, 1534/35 », in Franz Petri (éd.), Der Raum Westfalen 6. Fortschritte der Forschung und Schlubbilanz I, Münster, Aschendorff, p. 277-422.

KLÖTZER Ralf, 1992, Die Täuferherrschaft von Münster. Stadtreformation und Welterneuerung, Münster, Aschendorff.

KLUGE Dietrich, 1975, « Die Vorbereitung der Täuferherrschaft in Münster », Jahrbuch für Westfälische Kirchengeschichte, 68, p. 23-38.

KOBELT-GROCH Marion, 1992, « Frauen gegen Geistliche. Weiblicher Antiklerikalismus in frühreformatorischen und täuferischen Bewegungen », Mennonitische Geschichtsblätter, 49, p. 21-31.

KRAUS Hermann, 1993, Gütergemeinschaft und Polygamie - Die unmoralischen Wesensmerkmale des Kommunismus und jeder anderen Revolution, dargestellt am Täuferkommunismus - an einer 1534/35 im belagerten Münster 'endzeitlich' gelebten Menschheitsutopie, Fürth, Selbstverlag ( $2^{\mathrm{e}}$ éd.).

KURATSUKA Taira, 1985, « Gesamtgilde und Täufer : Der Radikalisierungsprozeß in der reformatorischen Bewegung zum Täuferreich 1534/35 », Archiv für Reformationsgeschichte, 76, p. 231-270.

LAUBE Adolf (éd.), 1992, Flugschriften vom Bauernkrieg zum Täuferreich (1526-1535), Berlin, Akademie Verlag.

--, (éd.), 2000, Flugschriften gegen die Reformation (1525-1530), Berlin, Akademie Verlag.

MENIUS Justus, (1544) 1983, « Vom Geist der Wiedertäufer », in Robert Stupperich (éd.), Die Schriften der münsterischen Täufer und ihrer Gegner, III. Schriften von evangelischer Seite gegen die Täufer, Münster, Aschendorffsche Verlagsbuchhandlung, p. 245-248.

MIKAT Paul, 1988, Die Polygamiefrage in der frühen Neuzeit, Opladen, Westdeutscher Verlag. NIESERT Joseph, 1826, Münsterische Urkundensammlung. Bd. 1. Urkunden zur Geschichte der Münsterischen Wiedertäufer, Coesfeld, Wittneven.

PETERS Christian, 2004, « Bernhard Rothmann », in Religion in Geschichte und Gegenwart, Handwörterbuch für Theologie und Religionswissenschaft, 7, Tübingen, Mohr Siebeck.

RHEGIUS Urbanus, (1527) 1992, « Wider den neuen Tauforden », in Adolf Laube (éd.), Flugschriften vom Bauernkrieg zum Täuferreich (1526-1535), Berlin, Akademie Verlag, p. 1167-1248.

RICCEUR Paul, 1997, L'idéologie et l'utopie, Paris, Seuil.

ROPER Lyndal, 1991, « Sexual Utopianism in the German Reformation », The Journal of Ecclesiastical History, 42/3, p. 394-418. 
ROTHMANN Bernhard, (1533) 1970, «Bekenntnisse van beyden sacramenten, doepe vnde nachtmaele, der predicanten tho munster », in Robert Stupperich (éd.), Die Schriften der münsterischen Täufer und ihrer Gegner, I. Die Schriften Bernhard Rothmanns, Münster, Aschendorffsche Verlagsbuchhandlung, p. 139-195.

--, (1534a) 1970, « Bekenntnis des glaubens und lebens der gemein Christi zu Münster », in Robert Stupperich (éd.), Die Schriften der münsterischen Täufer und ihrer Gegner, I. Die Schriften Bernhard Rothmanns, Münster, Aschendorffsche Verlagsbuchhandlung, p. 195-208.

--, (1534b) 1970, « Restitution rechter und gesunder christlicher Lehre », in Robert Stupperich (éd.), Die Schriften der münsterischen Täufer und ihrer Gegner, I. Die Schriften Bernhard Rothmanns, Münster, Aschendorffsche Verlagsbuchhandlung, p. 208-284.

--, (1534c) 1970, « Bericht von der wrake », in Robert Stupperich (éd.), Die Schriften der münsterischen Täufer und ihrer Gegner, I. Die Schriften Bernhard Rothmanns, Münster, Aschendorffsche Verlagsbuchhandlung, p. 284-297.

SATTLER Michael, (1527) 1962, « Brüderliche Vereinigung etlicher Kinder Gottes », in Heinold Fast (éd.), Der linke Flügel der Reformation. Glaubenszeugnisse der Täufer, Spiritualisten, Schwärmer und Antitrinitarier, Bremen, Carl Schünemann Verlag, p. 60-71.

SCHILDT Maurice, 1982, "Ehe / Eherecht / Ehescheidung. VII - Reformationszeit », in Theologische Realenzyklopädie, IX, Berlin / New York, W. de Gruyter, p. 336-346.

SCHÖNEBAUM Herbert, 1919, Kommunismus im Reformationszeitalter: Humanismus, Reformatoren, Wiedertäufer, Bonn / Leipzig, Kurt Schroeder Verlag.

SCRIBE Eugène / MEYERBEER Giacomo, 1849, Le Prophète, Paris.

SIEBERT Susanne, 1995, « Bernhard Rothmann », in Biographisch-Bibliographisches Kirchenlexikon, VIII, Herzberg, Bautz, p. 825-827.

STAYER James M., 1980, «Vielweiberei als “innerweltliche Askese”. Neue Eheauffassungen in der Reformationszeit », Mennonitische Geschichtsblätter, 32, p. 24-41.

--, 2001, « Täufer / Täuferische Gemeinschaften I », in Theologische Realenzyklopädie XXXII, Berlin / New York, W. de Gruyter, p. 597-618.

--, 2002, « Unsichere Geschichte : Der Fall Münster 1534-1535. Aktuelle Probleme der Forschung ", Mennonitische Geschichtsblätter, 59, p. 63-78.

STUPPERICH Robert, 1958, Das münsterische Täufertum. Ergebnisse und Probleme der neueren Forschung, Münster, Aschendorffsche Verlags-buchhandlung.

--, (éd.), 1970, Die Schriften der münsterischen Täufer und ihrer Gegner, I. Die Schriften Bernhard Rothmanns, Münster, Aschendorffsche Verlags-buchhandlung.

--, (éd.), 1980, Die Schriften der münsterischen Täufer und ihrer Gegner, II. Schriften von katholischer Seite gegen die Täufer, Münster, Aschendorffsche Verlagsbuchhandlung.

--, (éd.), 1983, Die Schriften der münsterischen Täufer und ihrer Gegner, III. Schriften von evangelischer Seite gegen die Täufer, Münster, Aschendorffsche Verlagsbuchhandlung.

VOGLER Günter, 1981, « Das Täuferreich zu Münster im Spiegel der Flugschriften », in HansJoachim Köhler (éd.), Flugschriften als Massenmedium der Reformationszeit, Stuttgart, Klett-Cotta, p. 309-353.

VOLTAIRE (1756) 1963, Essai sur les mœurs et l'esprit des nations, Paris, Garnier. 
WILLIAMS George Huntston, 1962, The Radical Reformation, Philadelphia, The Westminster Press ( $3^{\mathrm{e}}$ éd., 1992, Kirksville, Mo., The Sixteenth Century Journal Publ.).

WOLGAST Eike, 1978, « Herrschaftsorganisation und Herrschaftskrisen im Täuferreich von Münster 1534/35 ", Archiv für Reformationsgeschichte, 69, p. 179-201.

WUNDER Heide, 2001, « Frauen in der Reformation : Rezeptions und historiographiegeschichtliche

Überlegungen », Archiv für Reformations-geschichte, 92, p. 302-320.

YOURCENAR Marguerite, 1968, L'Eeuvre au noir, Paris, Gallimard.

ZIMMERLI-WITSCHI Alice, 1981, Frauen in der Reformationszeit, Zurich, Thèse de Doctorat.

ZUR MÜHLEN Karl-Heinz, 2001, « Taufe V - Reformationszeit », in Theologische Realenzyklopädie

XXXII, Berlin / New York, W. de Gruyter, p. 701-710.

\section{NOTES}

1. Rothmann (1534b) 1970.

2. Parmi une littérature assez abondante sur le sujet : Stayer 2001 et Goertz 1988.

3. Entre autres témoignages, voir la justification de la condamnation et de l'exécution de Balthasar Hubmaier par Johannes Fabri, publiée au printemps de 1528 : Fabri (1528a) 1992, en particulier p. 1589-1590. Fabri reprend cette argumentation dans ses sermons contre les anabaptistes prononcés en avril 1528 en Moravie et publiés par la suite : Fabri (1528b) 2000, en particulier p. 673. Voir également Bullinger (1560) 1983.

4. La signification du baptême est, elle aussi, très diverse suivant les courants : Zur Mühlen 2001.

5. Stayer $2001: 598$. Stupperich $1958: 11$.

6. Deppermann \& al. 1975.

7. Fast 1962.

8. Williams 1962.

9. Laube 1992 : 587 sqq. Ce rappel est volontairement schématique, l'anabaptisme ne se réduit évidemment pas à ces quatre variantes.

10. Sattler (1527) 1962. Michael Sattler était bénédictin, marié à une béguine après avoir quitté le couvent, il se fait baptiser (sans doute) à Zurich en 1525. Il dirige le premier synode des anabaptistes en 1527, à l'issue duquel est rédigée cette "Confession ». Arrêté à Horb, dans le sud de l'Allemagne, où il devait prendre la responsabilité de la communauté anabaptiste, il est condamné à mort le 19 mai 1527 et exécuté le 20 (ou 21) mai. Cf. Heinz 1995.

11. Né vers 1485 , il est exécuté à Vienne en mars 1528 . Théologien, réformateur de la ville de Waldshut en 1524, il fut par la suite l'un des premiers « théoriciens » de l'anabaptisme, qu'il y implanta à partir de 1525 et qu'il transplanta à partir de l'été 1526 à Nicolsbourg (Moravie, aujourd'hui : Mikulov, en République tchèque). Il s'est opposé à Huldrych Zwingli sur la conception du baptême, et à Hans Hut en particulier en ce qui concerne l'usage des armes et la participation à l'exercice du pouvoir et à la fiscalité. Cf. Bautz 1990b.

12. Il meurt en prison en décembre 1527 , avant la fin de son procès.

13. Né à la fin du XV siècle, il meurt en 1543 à Strasbourg où il est incarcéré depuis dix ans. Ancien artisan fourreur, il est prédicateur évangélique à partir de 1523 dans un certain nombre de villes autour de la Baltique, mais, à partir de 1525, est désavoué par 
les luthériens qui lui reprochent ses positions millénaristes et sa conception de l'eucharistie. Il séjourne à Strasbourg en 1529-1530, où il est en contact avec les anabaptistes. C'est lui qui propagera ce mouvement, dans sa conception personnelle, en Frise orientale et en Hollande. Cf. Bautz 1990c. Plus détaillé : Deppermann 1979.

14. Klaus Deppermann rappelle que la pensée de Melchior Hoffmann a donné naissance en Hollande à deux branches, l'une résolument pacifiste - les futurs mennonites -, l'autre, reprenant entre autres les annonces insistantes du « jour de la colère » où tous les « curés » seront exterminés et l'affirmation que les « saints parfaits » sont au-dessus des lois, ayant trouvé sa réalisation (éphémère) à Münster. Cf. Deppermann 1979 : 31 . 15. Né en 1495, on perd sa trace après la prise de Münster. Ordonné prêtre en 1529, il est vicaire à l'église Saint-Maurice, dans les faubourgs de Münster. Il passe de positions traditionnelles à l'évangélisme, puis au zwinglianisme dans sa conception de l'eucharistie, avant de défendre l'anabaptisme - et est présenté pour cette raison par James M. Stayer comme " élément imprévisible en matière de théologie » (Stayer 2001 : 608). Prédicateur de talent, seul véritable théologien dans la communauté de Münster, il en sera le porte-parole. Cf. Siebert 1995 ; Stupperich 1970 : XI-XVII ; Detmer 1904 ; Peters 2004.

16. Rothmann (1533) 1970.

17. Stupperich $1970: 129$ sq. et 138 . Le projet de règlement ecclésiastique est daté du mois d'avril ; soumis pour expertise aux théologiens de Marburg (luthériens), il fait l'objet d'un certain nombre de critiques portant notamment sur la conception de l'eucharistie et du baptême, critiques auxquelles Bernhard Rothmann répond au mois de juillet. Ce dernier texte servira de base à la dispute du mois d'août. Robert Stupperich mentionne les efforts du Conseil pour expulser les « prédicateurs de Wassenberg ", dont Heinrich Roll, comme faisant suite à cette dispute, et cite un arrêté d'expulsion du 10 décembre suivi à la fin du mois d'une révolte menée par Heinrich Roll leur permettant de se ré-installer à Münster. Voir également Kuratsuka 1985 : 252 qui propose une chronologie et une interprétation des faits différentes.

18. Adeptes de l'anabaptisme dans la version élaborée par Melchior Hoffmann - cf. note 13 supra.

19. 1500+-/1534. Ancien boulanger, ancien disciple de Melchior Hoffmann, dont il s'inspire, mais en revendiquant la validité immédiate des révélations et visions. C'est lui qui aurait envoyé Johann Bockelson (cf. infra) à Münster. Cf. Bernet 2003. Se considérant comme le nouvel Enoch, $2^{\mathrm{e}}$ prophète témoin de l'apocalypse, ce n'est pas à Strasbourg, mais à Münster qu'il place la Nouvelle Jérusalem. Cf. Stayer 2001: 608 . 20. Wolgast 1978 : 179-180 cit. in Fischer 2004 : § 15 note 29.

21. 27 février 1534. Cf. Gresbeck $1853: 19-21$. Cet événement est présenté comme " sanctification » de la communauté par Bernhard Rothmann - cf. Rothmann (1534b) $1970: 279-282$. Cette idée est reprise en ces termes au début de son traité suivant (« Récit de la colère [divine] »), daté de décembre 1534 - cf. Rothmann (1534c) 1970 : 285.

22. Rothmann (1534b) $1970: 256$ (chapitre 12); Gresbeck 1853 : 32-34.

23. Rothmann (1534b) $1970: 221$ (chapitre 2); Gresbeck $1853:$ 46-47.

24. Né en 1509, Jan Bockelson (/ Beukelsz / Jean de Leyde) était tailleur de formation ; il avait été baptisé par Jan Matthijs en 1533 et envoyé par celui-ci comme apôtre à Münster pour soutenir la cause anabaptiste. Arrêté lors de la chute de Münster, il est exécuté en janvier 1536. Cf. Bautz 1990a; Detmer 1903. Sur la mort de Jan Matthijs et sa succession : cf. Gresbeck $1853: 38-42$. 
25. Fischer 2004 : $§ 11,24,55$; Stayer 2001 : 605 : « nouveau Roi David »; Gresbeck 1853 : 35-36 et 82-86; Rothmann (1534b) $1970: 278$.

26. Les repas sont pris en commun avec prédication quotidienne : Gresbeck 1853 : 34-35 ; il est interdit de fermer les portes des logements : Gresbeck $1853: 47$; les mariages contractés avant le changement de régime doivent être renouvelés : Gresbeck 1853 : 71 ; les rues, places et portes de la ville changent de nom : Gresbeck 1853 : 154-155, les enfants reçoivent dorénavant leur nom du roi : Gresbeck 1853 : 156. 27. Gresbeck 1853 : 59-73. Pour être plus précis, il s'agit exclusivement de la polygynie, la polyandrie étant passible de la peine de mort - cf. Fischer $2004: \S 42$.

28. Gresbeck $1853:$ 73-79.

29. Stupperich $1958: 21$.

30. Stupperich $1970: 405-447$; Gresbeck $1853: 120-122$.

31. Stupperich $1970: 209$; Stayer $1980: 33$.

32. Stupperich 1980 ; Stupperich 1983.

33. Stupperich $1970: 209$.

34. Ac 3,19 sqq. : annonce de la restauration universelle et du renouvellement de toute la création ; Ac 15,16: promesse du retour du Christ et de la reconstruction du monde. 35. Chapitre 1.

36. Sa juste interprétation : chapitre 2, la relation entre Ancien et Nouveau Testament(s) : chapitre 3, l'enseignement du Christ : chapitre 3, le salut par le Christ : chapitre 5, le respect des préceptes divins et les œuvres : chapitre 9.

37. Chapitre 4.

38. Chapitre 7.

39. Chapitre 8.

40. Respectivement chapitres 10 et 11 .

41. « van leyfliker gemeinschup der Hilligen » : « De la communauté physique (/ charnelle) des saints ", c'est-à-dire la communauté de Münster : chapitre 12.

42. Chapitre 13.

43. Chapitre 17.

44. Chapitre 18.

45. Chapitres 14,15 et 16 .

46. « Van den rechten vnde christliken Ehestande. » Chapitre 14 - Rothmann (1534b) $1970: 258$.

47. Gn 1,27 sq., cité ici dans la traduction de la Bible de Jérusalem.

48. « [...] Wy hebben hyr beuoerns gesacht, wat de Eestandt sy, nemptlick, Dat de Ehestaet sy eyne voereinige des mans vnde wyues, den segen Gades tho syner eer vnde na sinen willen thouorkrigen, wo dan dar tho de mensche van Godt geschapen ys, vnnde einen beuel entfangen hefft. Gene. 1 [28] [...] Godt segenet den menschen vnd maket en fruchtbar, gifft em dar beneuen ein beuel, wo he sulcker gaue gebrucken sal, [...]»- Rothmann (1534b) $1970: 260$.

49. Hé 13,4, «[...] Car Dieu jugera fornicateurs et adultères.[...] » (trad. : cf. note 47 supra).

50. « [...] want eth hefft geinen wabdom, vnd och noch vmme ander saken mer. [...]»Rothmann (1534b) $1970: 261$.

51. «[... ] Thom derden ys de lesterlike vnde bestlike gebruick vorbaden, welck tegen de natur ys. [...]» - Rothmann (1534b) $1970: 261$. 
52. «[...] Thom veerden worth eth ock vor sunde gherekent, so yummant ein weeklinck ys vnde yn sick gebrant woert, [1 Cor. 7,9] offte durch den slaep sick verunreniget, [...] » - Rothmann (1534b) $1970: 261$.

53. « [...] Dyt ys dan, dat Paulus secht [... cit. : Hé 13,4], wanner man vnd wyff beide yn Gades fruechten vnd reinen gelouen staen, [...] Hyruth versteestu nu wal, [...] dat der haluen geine rechte Ehe wesen kan, dan alleine by den rechtgeloeuuigen, [...]»Rothmann (1534b) $1970: 261$.

54. « [...] vnd war die Ehe, dat ys Gades beuel yn der ehe nicht geholden wort, dar ys ehebreckerye und ydel horerye, [...] Vnd also ys Gades wille gewesen und ysset noch, dat welcke yn Christum tho dusser tyt gedoept geworden, ock yn dem ehestate moesten vernyet werden, up dat se gewasschen van allen sunden, allenthaluen vortan reyn wanderen moechten. [...]»- Rothmann (1534b) 1970 : 262. Cf. également Gresbeck 1853 : 62 et 71.

55. «Van Behoer vnd Herlichkeit des mans in der Ehe », chapitre 15 - Rothmann (1534b) 1970 : 262. "Behoeren" : lat. convenire, pertinere (DWB I, p. 1342). La traduction de ce terme par "rechte", c'est-à-dire "droits" (cf. Kraus 1993 : 32) est inexacte compte tenu de l'usage récurrent qui est fait du verbe et du substantif dans ces trois chapitres entre autres. Elle est anachronique par rapport au contexte et ne peut que résulter d'une lecture trop rapide, voire d'une interprétation erronée de ces têtes de chapitres. Le terme « Herlichkeit » est plus délicat à traduire; il évoque le statut du maître, du seigneur, la splendeur, la majesté, la grandeur, la gloire. C'est le terme consacré dans la Bible allemande en association avec Yahvé, Dieu, Christ, Salomon, par exemple. Cf. également Gresbeck 1853 : 68-69 et Klötzer $1992: 116$.

56. « [...] vnd dat he nicht mer yummandt anders en hoer, vp dat he nicht bedrogen werde, als do he dat wyff horde. [...] »- Rothmann (1534b) 1970 : 262, chapitre 15. 57. " [...] want als dan hefft he macht van Gade, ein heer tho wesen oeuer syn frouwe, gleick als Godt ein Heer oeuer em is.[...] » - Rothmann (1534b) 1970 : 263, chapitre 15. 58. «[...] de getzirdt sy mit gades fruchten vnd mit einen stillen und sachtmoedigen geiste.[...] »- Rothmann (1534b) $1970: 263$, chapitre 15.

59. « ein herr vnd ein hoeubt » - Rothmann (1534b) $1970: 263$; cette partie de l'argumentation est adossée à la formulation de l'Épître aux Éphésiens (Éph. 5,23 sqq.), le terme « das Haupt » signifiant à la fois le chef et la tête : la relation entre l'homme et la femme est la même que celle qui existe entre le Christ et son Église (/ sa communauté), le premier en est la tête, la seconde le corps / les membres. Cette argumentation est répétée dans le chapitre suivant.

60. « moitwillig » - c'est-à-dire si sa volonté est trop affirmée - Rothmann (1534b) 1970 : 264.

61. «[...] Dan angesein de herlike fryheit des mans in der Ehe vorloren ys und gans vorduestert, so ys de man dar mede seer beswert, unde so he der fryheit vnuorstendich blifft vnde der nicht en gebruket, ysset vnmoegelick, dat he de sunde vnd schande der vnreinicheit entflein moege. [...] Nu wal an dan, de fryheit des mans in der Ehe ys, dat he wal mer dan ein frouwe tho gelike Ehelick hebben mach, [...] »- Rothmann (1534b) $1970: 264$.

62. «[...] Hyrumme ys lichtlick tho mercken, dat den manne sulcker fryheit van noeden sy, sal he der sunde entlopen, want [...] » - ce qui suit est un rappel des interdits présentés au chapitre précédent : $c f$. ci-dessus, chapitre 14. Rothmann (1534b) 1970 : 265.

63. « [...] na siner kuescher notdrufft »- Rothmann (1534b) $1970: 268$. 
64. « [...] Die wyuer hebben vast allenthaluen de herrschappye, vnd leiden de menner als men de baren leidet, vnd alle werlt ys in ehebreckerie, vnreinicheiten vnd horerye vorsoppen.[...]» - Rothmann (1534b) 1970 : 268, chapitre 15.

65.« Van Behoer vnd Vnderdanichkeit der Frouwen in der Ehe » - Rothmann (1534b) 1970 : 268, chapitre 16.

66. " [...] want eth myt den frouwen dus lange ock gans verkert ys gewesen. [...]» Rothmann (1534b) 1970 : 268.

67. «[...] dit segge wy darumme, want welcke nicht frouwelick en syn, moegen den man in der Ehe in natuerliken gebruck nicht deinen, wo wal se anders yn leyffden vnd gehorsam eins myt eren manne blifft, vnde den manne allike wal als eren hoeuede vnde Heren vnderworpen bliuen. [...]» - Rothmann (1534b) 1970 : 268. Cette précision est rappelée à la fin du chapitre, p. 269 ; elle se traduira, dans les faits, par l'obligation faite aux femmes relevant de cette catégorie de se trouver un "protecteur". $C f$. Gresbeck 1853 : 68-69; Fischer 2004 : § 49.

68. « [...] also de frouwe eren man, vnde dat sunder allen murren vnde wedersprecken, als eren heren, [...] immer vor Augen haben [...] »- Rothmann (1534b) $1970: 269$, chapitre 16.

69. « [...] das hoeuet vnde de gebeider oeuer all ys Godt almechtig, [...] vnd vort, de man ys das hoeuet der frouwen [...]» - Rothmann (1534b) 1970 : 268-269.

70. « [...] also hebben ock de frouwen nicht tho regeren, dan my stillicheit gehorsam tho sein. [...]»- Rothmann (1534b) $1970: 269$.

71. « [...] oeuerst ys hoch van noeden, dat sick de menner recht yegen Christum holden, und als dan, wyuer, de nu vast allenthaluen de brock an hebben, in rechten vnd geboerliken gehorsam boegen, [...] » - Rothmann (1534b) 1970 : 269.

72. « [...] de menner sult nicht lenger wyuer syn, [...]»- Rothmann (1534b) 1970 : 269.

73. Jr 33,1 sqq.

74. « [...] so hefft he [Gott] nu by vns alle wyuer in gehorsam der menner gestalt, dat se alle so wal yunck als olt von dem manne durch Gades wort sick moeten regeren laten.

[...], » - Rothmann (1534b) $1970: 269$.

75. « [...] Ein yder wake vp. [...]» - Rothmann (1534b) $1970: 269$.

76. On trouve des accents similaires dès la fin du $\mathrm{XV}^{\mathrm{e}}$ siècle, notamment dans la Nef des Fous, de Sébastian Brant, ou un peu plus tard sous la plume d'Érasme, et dans le premier tiers du XVI ${ }^{\mathrm{e}}$ siècle. La position inverse, faisant de la femme « l'égale » de l'homme est extrêmement minoritaire : présente « en filigrane » chez certains anabaptistes et chez certains théologiens évangélistes, c'est chez Martin Bucer, dans ses réflexions sur le mariage, qu'elle sera le plus affirmée - cf. Schildt $1982: 341-343 ; c f$. également Stayer $1980: 25$ sq.

77. Hennig 1983 : 29-30.

78. Gresbeck $1853: 73-79$. Cet événement est mentionné également par Philippe de Hesse, Urbanus Rhegius (cf. Stupperich 1983), et Dorpius (1536) 1992 : 1675.

79. Gresbeck 1853 : 63-68; Fischer $2004: \S 81$.

80. «Öffentliche Verordnung der zwölf Ältesten » - Fischer 2004 : § 30 sqq.

81. Fischer 2004 : § 36, qui se fonde sur Kessenbroick (1575) 1900.

82. Stupperich $1970: 445$ sq.

83. Article 7 - Stupperich $1970: 445$.

84. Article 15 - Stupperich $1970: 446$.

85. Article 13 - Stupperich $1970: 445$. 
86. Argument récurrent depuis 1525 environ jusqu'à nos jours, puisqu'il réapparaît régulièrement, sans commentaire spécifique - $c f$. Schönebaum $1919: 21,25 s q ., 40$; Chaix $2002: 299$ entre autres exemples. Au seizième siècle, ils sont accusés de " nicolaïsme ", ou de pratiquer des « noces nicolaïtes ».

87. et un peu plus d'un millier d'enfants. Les chiffres sont cités ici d'après l'étude de Carsten Fischer (Fischer 2004 : § 44), qui les arrondit en se référant à Karl-Heinz Kirchhoff (Kirchhoff 1989 : 392 sqq.), qui dénombre 4800 femmes, 1755 hommes, et 1200 enfants. L'évaluation de la population totale de Münster, 8 à 9000 personnes, semble être communément acceptée dans la recherche. Cf. également : Stayer 2001 : 605.

88. Les sources ne sont pas très explicites à cet égard ; l'afflux d'anabaptistes venus de Frise, de Hollande et de Westphalie n'est pas contesté, James Stayer (Stayer $2001: 605$ ) évalue leur nombre à environ 2500 personnes, remplaçant les quelque 2000 habitants expulsés. Pour la postérité, cet élément est ré-interprété dans un sens négatif, puisque ces nouveaux arrivants sont pour ces auteurs des femmes ayant délaissé leurs époux et des hommes ayant abandonné femmes et enfants, signe évident de leur piètre moralité - cf. entre autres Bullinger (1560) $1983: 253$.

89. Gresbeck $1853: 62$ sq. ; Fischer 2004 : § 46.

90. Gresbeck $1853: 13-21$; Dorpius (1536) $1992: 1672$.

91. Hennig $1983: 31$ sq. Il se fonde sur les thèses présentées par James M. Stayer in Stayer 1980.

92. Kluge 1975.

93. Hennig $1983: 38$; voir également Roper 1991 et Klötzer $1992: 101$.

94. Celle-ci n'est d'une part pas réellement homogène au départ, mais soudée entre autres par un régime de terreur. D'autre part, les activités traditionnelles - commerce, artisanat, agriculture et élevage - sont soit interdites, soit très réduites, à la fois du fait de l'abolition de la propriété privée et des échanges monétaires, et à cause du siège qui limite nécessairement les échanges ainsi que le territoire disponible ; restent les activités liées à la défense de la ville, au contrôle de la population, les repas pris en commun perdant peu à peu de leur importance au fur et à mesure que les ressources s'épuisent et que la famine s'installe ; le temps libéré par la disparition des activités traditionnelles est sans doute utilisé à d'autres fins ; c'est donc probablement ce même souci - occuper la population pour la contrôler - qui préside à la réintroduction du jeu, de la musique, de la danse et du théâtre alors que ces activités étaient jusqu'alors interdites. Cf. Gresbeck 1853: 54 sqq., 131-140, 168. Parmi les comportements sexuels "déviants", on trouve mention dans le récit de Gresbeck de faits qui, transposés dans la terminologie d'aujourd'hui, correspondraient à du harcèlement (Gresbeck $1853: 63$ ), des viols (Gresbeck 1853: 62), des viols sur mineures (fillettes à partir de 11 ans Gresbeck $1853: 62$ et 72$)$.

95. Hennig 1983 : 38 ; Stayer $2001: 610$. Dans son article « Vielweiberei », James M. Stayer présente la polygamie et l'hostilité à l'égard des femmes

(«Frauenfeindlichkeit ») comme les traits marquants de cet ascétisme radical qu'il voit concrétisé à Münster (Stayer 1980 : 34).

96. Hennig $1983: 39$.

97. Cf. Rothmann (1534b) 1970 : chapitre 16.

98. Schönebaum $1919: 36$, Stayer 2002.

99. Terminée en mai 1525 par l'écrasement des insurgés après avoir fait, selon le camp catholique, environ cent mille morts - $c f$. Laube $2000: 22$. Ce mouvement est considéré par l'historiographie allemande en particulier comme la première révolution en 
territoire germanique, puisqu'il s'agissait du premier mouvement d'insurrection supraterritorial touchant à la fois le monde urbain et le monde rural, et disposant d'un programme revendiquant des réformes dans les domaines politique, social et religieux - cf. Blickle 1993. Ce mouvement naît dans le sillage de la Réforme et pour les catholiques, la responsabilité en revient aux luthériens, qui s'en défendront en mettant en avant leur respect de l'ordre et de l'autorité, et en accusant les « Schwärmer », principalement Andreas Carlstadt et Thomas Müntzer, auxquels ils assimileront progressivement les anabaptistes - cf. Dejeumont 2002.

100. Parmi les portraits les plus complets, il faut citer du côté luthérien le pamphlet d'Urbanus Rhegius, responsable de la répression des anabaptistes à Augsbourg : "Contre le nouvel ordre des baptistes ", publié en 1527 - Rhegius (1527) 1992 - et du côté catholique, ceux de Johannes Fabri, impliqué dans la condamnation de Balthasar Hubmaier à Vienne et la répression des anabaptistes en Moravie - Fabri (1528a) 1992 et Fabri (1528b) 2000. Dans ses « sermons contre les anabaptistes », Fabri cite le texte de Rhegius mentionné ci-dessus.

101. Philippe Mélanchthon, Urbanus Rhegius, Philippe de Hesse - cf. Stupperich 1983. 102.Adolf Laube mentionne plus d'une douzaine de titres - Laube $1992: 1506$ sq. Günter Vogler en avait identifié une quinzaine, tous publiés en 1535-1536 - Vogler 1981. Sigrun Haude en ajoute deux - Haude $2000: 18$.

103. Dorpius (1536) 1983 et 1992 ; Stupperich $1983: 220$; Laube $1992: 1683$.

104. Laube $1992: 1682$ et 1730 . Tandis que les deux premiers ont joué un rôle majeur dans les événements, il semble que le dernier ait expié à la place de Bernhard Rothmann, dont la trace s'est perdue après la chute de Münster - Klötzer 1992 : 137 . 105. Menius (1544) 1983 ; cette édition ne présente que les passages consacrés aux événements de Münster, le texte original étant plus volumineux.

106. Menius (1544) $1983: 248$.

107. Cette assimilation apparaît déjà dans un texte anonyme de $1535:$ « Neue Zeitung die Wiedertäufer zu Münster belangend » - cf. Laube $1992: 1631$ - mais qui n'aura pas la même diffusion que l'ouvrage de Menius.

108. Bullinger (1560) $1983: 254,258,263,264$.

109. Engels $1960: 356$ sqq.

110. Ricœur 1997 : 363.

111. Rothmann (1534a) $1970: 205$.

112. Ou présentées comme telles - cf. Roper 1991.

113. Zimmerli-Witschi 1981 : 147-151 et 154-157 - elle aussi établit un lien direct entre communauté des biens et liberté sexuelle. Cf. également : Haas $1977: 69$; Fast 1962 : XVII.

114. Cornelius 1853 ; voir également : Cornelius : 1855 et 1860.

115. Cornelius 1853 : LXVIII sqq.

116. Engels $1960: 342-358$, chapitre II.

117. Chaix $2002: 299$.

118. À titre d'exemple : Kraus 1993.

119. 26 avril 1968, éditions Gallimard, Paris. Ce roman sera le Prix Fémina de l'année.

120. Haddad $1990: 104$.

121. Cit. in Hennig $1983: 28$ et 41 sq., note 33 ; pour relativiser ces positions, voir également les conclusions en demi-teintes proposées par James M. Stayer (Stayer 2001 : 614), et celles, plus tranchées, de Nicole Grochowina (Grochowina 1999) et de Marion Kobelt-Groch (Kobelt-Groch 1992 : 29). 
122. Rothmann (1534b) $1970: 250$ et 252, chapitre 10 .

123. Cf. Conrad / Michalik 1999:6 - ces deux historiennes font remarquer la rareté de ces sources. Alice Zimmerli-Witschi fait la même remarque et indique que la plupart des informations relatives aux femmes anabaptistes sont à chercher dans les rapports d'interrogatoires faisant suite à leur arrestation (Zimmerli-Witschi 1981: 2 et 133) ; Heide Wunder et Nicole Grochowina parviennent à des conclusions similaires (Wunder 2001 : 311-314 ; Grochowina 1999 : 101).

124. Mentionnées notamment in : Niesert $1826: 155$ et 165.

125. Rothmann (1534b) $1970: 281$, chapitre $18:$ : [...] eth Propheterde allent, wat dar was, ock de kinder van seuen yaren, [...]».

126. Telle semble être l'opinion de Voltaire à leur égard - Voltaire (1756) 1963 : chapitre CXXXII - et celle d'Eugène Scribe, librettiste de l'opéra de G. Meyerbeer intitulé Le Prophète, créé à Paris en 1849. Scribe cite certes Voltaire au début de son livret, mais dans celui-ci, les anabaptistes sont présentés comme des brigands assassins, manipulateurs et traîtres, utilisant le (faux) prophète Jean de Leyde pris entre sa fiancée, sa mère et les structures sociales. L'histoire de Münster fournit ici l'argument de départ, mais est traitée avec une très grande liberté.

127. Cf. pour Münster : Klötzer $1992: 16$ sqq.

128. Théodore de Bèze consacrera un traité à cette question, réédité à de nombreuses reprises à partir de 1568 (Bèze, 1568); cf. également Mikat 1988.

129. Cf. Bourdieu 1998.

\section{RÉSUMÉS}

La ville de Münster, en Westphalie, abrita en 1534-35 une communauté anabaptiste dont on ne connaît souvent que certains traits : son écrasement par les forces « de la réaction ", sa réforme du mariage qui défraya la chronique ou la prépondérance des femmes dans sa population..., parfois associés dans l'image d'une société révolutionnaire, libertaire, dans lesquelles les femmes avaient un grand rôle - qu'en est-il de ces idées reçues? Après un bref rappel de ce qu'est l'anabaptisme et de ce que fut cet épisode dans l'histoire de la Réforme, l'auteur analyse le texte justifiant l'instauration de la polygamie, ainsi que son traitement par l'historiographie et tente par ce biais de rétablir les faits dans leur contexte.

In 1534-35, the city of Münster in Westphalia was ruled by Anabaptists. For later generations, this episode has often been reduced to a few aspects, e.g. their defeat by the "forces of reaction', their scandalous reform of marriage, or the large proportion of women in the community. These are often combined to create the image of a revolutionary and 'women-friendly' community. After a brief survey of the history of Anabaptism and a brief presentation of the changes which occurred in Münster, the author analyses the text which justified the practice of polygamy, as well as the historiographical treatment of these events, and thus tries to situate the events in their context. 


\section{INDEX}

Mots-clés : anabaptisme, histoire de la Réforme, mariage, Münster, polygamie, utopie Index géographique : Münster

\section{AUTEUR}

\section{CATHERINE DEJEUMONT}

Catherine DEJEUMONT, maître de conférences à l'Université de Paris X - Nanterre, est germaniste, spécialiste du « frühneuhochdeutsch », et titulaire d'un doctorat sur l'Étude comparative de traductions allemandes du Nouveau Testament (1989). Ses recherches portent sur l'analyse du discours dans les pamphlets liés à l'émergence de la Réforme en Allemagne. Elle a notamment publié : « Dialogue entre un maire et un curé ... » (1521 - Martin Bucer (?)) : Traduction et commentaire, p. 7-43, in Marianne Ruel Robins (dir.) : Paroles d'Evangile (quatre pamphlets allemands des années 1520 : Martin Bucer, Christian Entfelder, Argula von Grumbach, Andreas Karlstadt), Paris, Presses de la Sorbonne, 1996, p. 7-43 ; « Le Septembertestament (1522) - Martin Luther et la langue allemande ", in Jean-Marie Valentin (dir.) : Luther et la Réforme - du Commentaire de l'Épître aux Romains à la Messe allemande, Paris, Desjonquères, 2001, p. 169-187 ; " "Schwärmer, Geist, Täufer, Ketzer" : de l'allié au criminel (1522-1550) », Bulletin de la Société de l'Histoire du Protestantisme Français, Tome 148/1, 2002, p. 21-44 ; «Commune, Communauté : Martin Luther et le Communalisme ", Journal de la Renaissance, Volume II, 2004, p. 287-296 ; «Flugschriften et Réforme : Frère Henri et la grand-mère (1523) », in André Combes \& Françoise Knopper (dir.) : L'opinion publique dans les pays de langue allemande, Paris, L'Harmattan, 2006 (sous presse) p. 19-38. 